\title{
ALMOST CONVERGENCE AND ALMOST SUMMABILITY
}

\author{
EKREM SAVAS
}

Summary. The purpose of this paper is to introduce and discuss the spaces of almost summable sequences. Also some matrix transformations have been characterized.

\section{Introduction}

Let $s$ be the set of all sequences with real or complex terms and let $\ell_{\infty, c}$ and $c_{0}$ denote, respectively, the Banach spaces of bounded, convergent and null sequences $x=\left(x_{k}\right)$ with the usual norm $\|x\|=\sup _{k}\left|x_{k}\right|$.

Let $D$ be the shift operator on s,i.e.,

$$
D\left(\left(x_{k}\right)\right)=\left(x_{k+1}\right) \text {. }
$$

It may be recalled that Banach limit $L$ is a nonegative linear functional on $\ell_{\infty}$ such that $L$ is invariant under the shift operator (i.e., $L(D x)=L(x)$ for all $x \in \ell_{\infty}$ ) and that $L(e)=1$ where $e=(1,1,1, \ldots),[1]$. A sequence $x \in \ell_{\infty}$ is said to be almost convergent if all Banach limits of $x$ coincide, [2].

Let $\hat{c}$ denote the set of all almost convergent sequences.

For any sequence $x$, we write

$$
t_{m n}=t_{m n}(x)=\frac{1}{m+1} \sum_{i=1}^{m} x_{n+i} .
$$

Lorentz [2] established the following result:

Theorem $\mathbb{A} . x \in \hat{c}$ if and only if $t_{m n}(x)$ tends to a limit as $m \rightarrow \infty$, uniformly in $n$.

Recently, some new sequence spaces which arose naturally from the concept of almost convergence have been introduced by Nanda [4]. If $\left(p_{m}\right)$ is a bounded sequence of positive real numbers, then we define (see, [4]),

$$
\begin{aligned}
& \bar{\ell}(p)=\left\{x: \sum_{m}\left|t_{m n}\right|^{p_{m}} \text { converges uniformly in } n\right\} \\
& \overline{\bar{\ell}}(p)=\left\{x: \sup _{n} \sum_{m}\left|t_{m n}\right|^{p_{m}}<\infty\right\} .
\end{aligned}
$$

Received June 13, 1989. 
(Here and afterwards summations without limits run from 1 to $\infty$ ). If $p_{m}=p$ for all $m$, we write $\bar{\ell}_{p}$ and $\overline{\bar{\ell}}_{p}$ for $\bar{\ell}(p)$ and $\overline{\bar{\ell}}(p)$ respectively. If $p_{m}=1$ we write $\bar{\ell}$ and $\overline{\bar{\ell}}$ for $\bar{\ell}_{p}$ and $\bar{\ell}_{p}$ respectively.

Let $A=\left(a_{n k}\right)$ be an infinite matrix of real or complex numbers. We write $A x=$ $\left(A_{n}(x)\right)$ if $A_{n}(x)=\Sigma_{k} a_{n k} x_{k}$ converges for each $n$. Let $X$ and $Y$ be any two nonempty subsets of $s$. If $\left(x_{k}\right) \in X$ implies that $A x=\left(A_{n}(x)\right) \in Y$, we say that $A$ defines a matrix transformation from $X$ into $Y$ and we denote it by $A: X \rightarrow Y$. By $(X, Y)$ we mean the class of matrices $A$ such that $A: X \rightarrow Y$.

\section{Almost summability.}

We define

$$
\begin{aligned}
(A, p) & =\left\{x: \sum_{n}\left|A_{n}(x)\right|^{p_{n}}<\infty\right\} \\
(A, p)_{\infty} & =\left\{x: \sup _{n}\left|A_{n}(x)\right|^{p_{n}}<\infty\right\} \\
(\hat{A}, p) & =\left\{x: \sum_{m}\left|t_{m n}(A x)\right|^{p_{m}} \text { converges uniformly in } n\right\} \\
(\hat{\hat{A}}, p) & =\left\{x: \sup _{n} \sum_{m}\left|t_{m n}(A x)\right|^{p_{m}}<\infty\right\}
\end{aligned}
$$

where

$$
t_{m n}(A x)=\sum_{k} a(n, k, m) x_{k}
$$

such that

$$
a(n, k, m)=\frac{1}{m+1} \sum_{i=0}^{m} a_{n+i, k} .
$$

If $p_{n}=p$ for all $n$ then we write $(A)_{p}$ and $(A)_{p}^{\infty}$ for $(A, p)$ and $(A, p)_{\infty}$ respectively. If $p=1$ we omit the suffix $p$ and write $(A)$. Note that $(A)$ denotes the set of all absolutely summable sequences. Similarly if $p_{m}=p$ for all $m$ we write $(\hat{A})_{p}$ and $\left(\widehat{\hat{A}}_{p}\right)$ for $(\hat{A}, p)$ and $(\widehat{\hat{A}}, p)$ respectively.

We have

Theorem 1. $(\hat{A}, p) \subset(\hat{\hat{A}}, p)$.

Proof. Let $x \in(\hat{A}, p)$. Then there is an integer $M$ such that

$$
\sum_{m \geq M}\left|\sum_{k} a(n, k, m) x_{k}\right|^{p_{m}} \leq 1 .
$$

Hence it is enough to show that, for fixed $m, \Sigma_{k} a(n, k, m) x_{k}$ is bounded. It follows from (2.1) that

$$
\left|\sum_{k} a(n, k, m) x_{k}\right| \leq 1 \text { for } m \geq M \text { and for all } n
$$


But if $m \geq 1$

$$
(m+1) \sum_{k} a(n, k, m) x_{k}-m \sum_{k} a(n, k, m-1) x_{k}=\sum_{k} a_{n+m, k}
$$

Hence for any fixed $m \geq M+1, \Sigma_{k} a_{n+m, k} x_{k}$ is bounded. Therefore $\Sigma_{k} a(n, k, m) x_{k}$ is bounded for all $m, n$ and this completes the proof.

Theorem 2. $(\hat{\hat{A}})_{p} \subset(A)_{\infty}$.

Proof. Note that

$$
\begin{aligned}
\sup _{m, n}\left|\sum_{k} a(n, k, m) x_{k}\right| & \leq \sup _{n}\left(\sum_{m}\left|\sum_{k} a(n, k, m) x_{k}\right|^{p}\right)^{1 / p} \\
\sup _{n, m}\left|\sum_{k} a(n, k, m) x_{k}\right| & \geq \sup _{n}\left|\sum_{k} a(n, k, 0) x_{k}\right|=\sup _{n}\left|\sum_{k} a_{n k} x_{k}\right| \\
\sup _{n, m}\left|\sum_{k} a(n, k, m) x_{k}\right| & =\sup _{n, m}\left|\sum_{k} \frac{1}{m+1} \sum_{i=0}^{m} a_{n+i, x_{k}}\right| \\
& \leq \sup _{n, i}\left|\sum_{k} a_{n+i, k} x_{k}\right| \sup _{m} \frac{\sum_{i=1}^{m} 1}{m+1} \\
& \leq \sup _{n, i}\left|\sum_{k} a_{n+i, k} x_{k}\right| \\
& =\sup _{n}\left|\sum_{k} a_{n k} x_{k}\right| .
\end{aligned}
$$

Now the result follows from (2.3), (2.4) and (2.5).

If $X$ is a linear space over the field $C$ then a paranorm on $X$ is a function $g: X \rightarrow R$ which satisfies the following axioms for $x, y \in X$,

$$
\begin{aligned}
g(0) & =0 \\
g(x) & =g(-x) \\
g(x+y) & \leq g(x)+g(y) \\
\lambda \longrightarrow \lambda_{0}, x & \longrightarrow x_{0} \text { imply } \lambda x \longrightarrow \lambda_{0} x_{0}
\end{aligned}
$$

where $\lambda, \lambda_{0} \in C$ and $x, x_{0} \in X$; in other words, $\left|\lambda-\lambda_{0}\right| \rightarrow 0, g\left(x-x_{0}\right) \rightarrow 0$ imply $g\left(\lambda x-\lambda_{0} x_{0}\right) \rightarrow 0$. A paranormed space is a linear space $X$ with a paranorm $g$ and is written as $(X, g)$.

Theorem 3. $(A, p)$ is linear topological space paranormed by

$$
f(x)=\left(\sum_{n}\left|\sum_{k} a_{n k} x_{k}\right|^{p_{n}}\right)^{1 / M}
$$


where $M=\max \left(1, \sup p_{n}\right) \cdot(\hat{A}, p)$ is paranormed by

$$
g(x)=\sup \left(\sum_{m}\left|\sum_{k} a(n, k, m) x_{k}\right|^{p_{m}}\right)^{1 / M}
$$

$(\hat{\hat{A}}, p)$ is paranormed by (2.6) if inf $p_{m}>0$. Also if inf $p_{n}>0$ then $(A, p)_{\infty}$ is paranormed by

$$
h(x)=\sup _{n}\left|\sum_{k} a_{n k} x_{k}\right|^{p_{n} / M} .
$$

Proof. Because of Theorem 1 (2.6) is meaningful for $x \in(\hat{A}, p)$. We consider only $(\hat{A}, p)$. It can be proved by "standart" arguments that $g$ is a paranorm on $(\hat{A}, p)$. As one step in the proof, we shall only show that for fixed $x, \lambda x \rightarrow 0$ as $\lambda \rightarrow 0$. If $x \in(\hat{A}, p)$, then given $\varepsilon>0$ there is an $M$ such that, for all $n$

$$
\sum_{m \geq M}\left|\sum_{k} a(n, k, m) x_{k}\right|^{p_{m}}<\varepsilon
$$

So if $0<\lambda \leq 1$, then

$$
\sum_{m \geq M}\left|\sum_{k} a(n, k, m) \lambda x_{k}\right|^{p_{m}} \leq \sum_{m \geq M}\left|\sum_{k} a(n, k, m) x_{k}\right|^{p_{m}}<\varepsilon
$$

and since, for fixed $M$,

$$
\sum_{m=0}^{M-1}\left|\sum_{k} a(n, k, m) \lambda x_{k}\right|^{p_{m}} \rightarrow 0
$$

as $\lambda \rightarrow 0$, this completes the proof.

Theorem 4. Let $0<p_{m} \leq q_{m}$, then $(\hat{A}, p) \subset(\hat{A}, q)$.

Proof. Let $x \in(A, p)$. then there is an integer $M$ such that (2.1) holds. Hence for $m \geq M\left|\Sigma_{k} a(n, k, m) x_{k}\right| \leq 1$. So that

$$
\left|\sum_{k} a(n, k, m) x_{k}\right|^{q_{m}} \leq\left|\sum_{k} a(n, k, m) x_{k}\right|^{p_{m}}
$$

and this completes the proof.

\section{Some matrix transformations}

In this section we characterize some matrix transformations 
Theorem 5. Let $b_{n k}>0$. If

$$
\sup _{n} \sum_{k}\left|a_{n k}\right|\left(b_{n k}\right)^{1 / p}<\infty
$$

and

$$
\sup _{k} \sum_{m}|a(n, k, m)|\left(b_{n k}\right)^{-1 / q}<\infty
$$

where $p^{-1}+q^{-1}=1$, then $A \in\left(\ell_{p}, \bar{\ell}_{p}\right)$.

Proof. We have by Hölder's inequality

$$
\begin{aligned}
\left|t_{m n}(A x)\right|^{p} & \leq\left(\sum_{k}|a(n, k, m)|\left(b_{n k}\right)^{1 / p}\right)^{p-1} \\
& \sum_{k}|a(n, k, m)|\left(b_{n k}\right)^{-1 / q}\left|x_{k}\right|^{p} .
\end{aligned}
$$

Hence

$$
\begin{aligned}
\sum_{m}\left|t_{m n}(A x)\right|^{p} & \leq\left(\sum_{k}|a(n, k, m)|\left(b_{n k}\right)^{1 / p}\right)^{p-1} \\
& \sum_{k}\left|x_{k}\right|^{p} \sum_{m}|a(n, k, m)|\left(b_{n k}\right)^{-1 / q}
\end{aligned}
$$

Also it follows from Lemma in [4] that (3.1) is equivalent to

$$
\sup _{n} \sum_{k}|a(n, k, m)|\left(b_{n k}\right)^{1 / p}<\infty .
$$

Now the result follows from $(3,2),(3.3)$ and (3.4).

Theorem 6. Let $1 \leq p<\infty$. Then $A \in\left(\ell_{\infty}, \bar{\ell}_{p}\right)$ if and only if

$$
\sum_{m}\left(\sum_{k}|a(n, k, m)|\right)^{p}<\infty, \text { uniformly in } n
$$

Proof. Sufficiency. Suppose that (3.5) holds and that $x \in \ell_{\infty}$. then

$$
\begin{aligned}
\sum_{m}\left|t_{m n}(A x)\right|^{p} & \leq \sum_{m}\left(\sum_{k}\left|a(n, k, m) x_{k}\right|\right)^{p} \\
& \leq\|x\|_{\infty}^{p} \sum_{m}\left(\sum_{k}|a(n, k, m)|\right)^{p} .
\end{aligned}
$$

Therefore $\Sigma_{m}\left|t_{m n}(A x)\right|^{p}$ converges uniformly in $n$ and so $A \in\left(\ell_{\infty}, \bar{\ell}_{p}\right)$. 
Necessity. Suppose that $A \in\left(\ell_{\infty}, \bar{\ell}_{p}\right)$ and that $x \in \ell_{\infty}$, Therefore

$$
q_{n}(x)=\left(\sum_{m}\left|t_{m n}(A x)\right|^{p}\right)^{1 / p}
$$

exists uniformly in $n$. Now $\left(q_{n}\right)$ is a sequence of continuous seminorms on $\ell_{\infty}$ such that $\sup _{n} q_{n}(2)<\infty$. Therefore by Banach-Steinhaus theorem ([3], p.114) there exists a constant $K$ such that

$$
q_{n}(x) \leq K \cdot\|x\| \quad\left(\forall n, \forall x \in \ell_{\infty}\right)
$$

Putting $x=\operatorname{sgn} a(n, k, m)$ in (3.6) we observe that (3.5) holds. this completes the proof.

\section{References.}

[1] Banach, S., Theorie des Operations linearies (Warszawa), (1932).

[2] Lorentz, G.G., "A contribution to the theory of divergent sequences", Acta Math. 80, 167-190, (1948).

[3] Maddox, I.J., Elements of Functional Analysis, cambridge University Press, (1970).

[4] Nanda, S., "Some paranormed sequence spaces", Rendiconti di Matematica (1), Vol. 4, serie VII, 11-19, (1984).

[5] Nanda, S., "Absolute almost convergence and absolute almost summability", Rendiconti di Mathematica dell sue appliczioni Perie VII-Vol.5, No.1-2, (1985).

Department of Mathematics, Firat University, ELAZIĞ/TURKEY. 